Pacific Journal of Mathematics

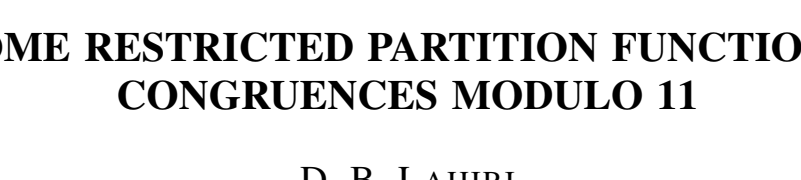




\title{
SOME RESTRICTED PARTITION FUNCTIONS: CONGRUENCES MODULO 11
}

\author{
D. B. LAHIRI
}

Ramanujan's congruences for the unrestricted partition function $p(n)$ with 5,7 and 11 as moduli can be shown to be equivalent to precisely similar congruences for some restricted partition functions of the type

$$
{ }_{r}^{t} p(n)
$$

where to determine the value of (1) we count all the unrestricted partitions of $n$ excepting those which contain any number of the forms $t n$ or $t n \pm r$ as a part. The purpose of the present paper is to deal with congruences modulo 11.

In [5] the author has established a number of congruences modulo 3 for (1) with certain selected values of $t$ and $r$. Functions of the type (1) are not new in number theory literature; for example, in the combinatorial interpretation of the famous Rogers-Ramanujan identities one finds

$$
{ }_{1}^{5} p(n), \quad{ }_{2}^{5} p(n)
$$

2. The final results. The restricted partition function (1) with $t=363$ and $r=121$ has a somewhat simpler interpretation. It is easily seen that this function counts the unrestricted partitions of $n$ excepting those which contain 121 or any multiple thereof as a part. We use the simpler notation

$$
{ }^{121} p(n)={ }_{0}^{121} p(n)={ }_{121}^{363} p(n),
$$

in the theorems to emphasize this interpretation.

The phrase 'for almost all values of $n$ ' appearing in Theorem 1 means that the number of integers $n \leqq N$ for which any specified congruence does not hold is $o(N)$. We assume ${ }_{r}^{t} p(m)$ to be 1 when $m=0$, and 0 when $m<0$.

THeOREM 1. For almost all values of $n$ the following congruences with respect to the modulus 11 hold. 


$$
\begin{aligned}
& { }^{121} p(n) \equiv 0, \\
& { }_{176}^{363} p(n) \equiv-{ }_{55^{3}}{ }^{2}(n-22) \text { ， } \\
& { }_{154} p(n) \equiv-{ }_{88}^{363} p(n-11) \text {, } \\
& { }_{143}^{363} p(n) \equiv-{ }^{363} 2^{p(n-33)} \text {, } \\
& { }_{77} p(n): \quad 363 p(n-11) \text {, } \\
& { }_{110}^{363} p(n) \equiv{ }_{11}^{363} p(n-33) .
\end{aligned}
$$

THEOREM 2. For all values of $n \geqq 0$

$$
{ }^{121} p(11 n+6) \equiv 0 \quad(\bmod 11),
$$

and more generally with $0 \leqq \lambda \leqq 16$

$$
{ }_{11 \lambda}^{363} p(11 n+6) \equiv 0 \quad(\bmod 11) .
$$

THEOREM 3. The following congruences modulo 11 are true for all values of $n \geqq 0$.

$$
\begin{aligned}
& -{ }_{176}^{363} p(11 n+10)-{ }_{55}^{363} p(11 n-12) \equiv 5^{121} p(11 n+5) \text {, } \\
& { }_{154}^{363} p(11 n+7)+{ }_{88}^{363} p(11 n-4) \equiv 5^{121} p(11 n+3) \text {, } \\
& { }_{143}^{363} p(11 n+4)+{ }_{22}^{363} p(11 n-29) \equiv 5^{121} p(11 n+1), \\
& { }_{77}^{363} p(11 n-2)-{ }_{44}^{363} p(11 n-13) \equiv 5^{121} p(11 n+8) ， \\
& -{ }_{110}^{363} p(11 n \quad)+{ }_{11}^{363} p(11 n-33) \equiv 5^{121} p(11 n+2) ， \\
& -{ }_{176}^{363} p(11 n+9)-{ }_{55}^{363} p(11 n-13) \equiv 5^{121} p(11 n+4) ， \\
& { }_{154}^{363} p(11 n+4)+{ }_{88}^{363} p(11 n-7) \equiv 5^{121} p(11 n \quad) \text {, } \\
& { }_{143}^{363} p(11 n+10)+{ }_{22}^{363} p(11 n-23) \equiv 5^{121} p(11 n+7) \text {, } \\
& { }_{77}^{363} p(11 n \quad) \quad-{ }_{44}^{363} p(11 n-11) \equiv 5^{121} p(11 n+10) ， \\
& -{ }_{110}^{363} p(11 n+7)+{ }_{11}^{363} p(11 n-26) \equiv 5^{121} p(11 n+9) .
\end{aligned}
$$


THEOREM 4. The following congruences with respect to the modulus 11 hold for all values of $n \geqq 0$.

$$
\begin{aligned}
& { }_{176}^{363} p(121 n+121)-{ }_{176}^{363} p(11 n+11) \\
& \equiv-{ }_{55}^{363} p(121 n+99)+{ }_{55}^{363} p(11 n-11) \text {, } \\
& { }_{154}^{363} p(121 n+120)-{ }_{154}^{363} p(11 n+10) \\
& \equiv-{ }_{88}^{363} p(121 n+109)+{ }_{88}^{363} p(11 n-1) \text {, } \\
& { }_{143}^{363} p(121 n+119)-{ }_{143}^{363} p(11 n+9) \\
& \equiv-{ }_{22}^{363} p(121 n+86)+{ }_{22}^{363} p(11 n-24) \text {, } \\
& { }_{77}^{363} p(121 n+106)-{ }_{77} p 3(11 n-4) \\
& \equiv{ }_{44}^{363} p(121 n+95)-{ }_{44}^{363} p(11 n-15), \\
& { }_{110}^{363} p(121 n+114)-{ }_{110}^{363} p(11 n+4) \\
& \equiv{ }_{11}^{363} p(121 n+81)-{ }_{11}^{363} p(11 n-29) .
\end{aligned}
$$

3. Notations and conventions. Ramanujan [8] defined

$$
\Phi_{r, s}(x)=\sum_{\alpha=1}^{\infty} \sum_{\beta=1}^{\infty} \alpha^{r} \beta^{s} x^{\alpha \beta}=\sum_{n=1}^{\infty} n^{r} \sigma_{s-r}(n) x^{n},
$$

where $\sigma_{k}(n)$ is, as usual, the sum of the kth powers of the divisors of $n$. The author has found it convenient to simplify the notation to $\Phi_{r, s},[3]$, and even to just $(r, s),[4]$, so that

$$
(r, s)=\sum_{n=1}^{\infty} n^{r} \sigma_{s-r}(n) x^{n} .
$$

The meanings of $f(x), u_{r}, v$ and $\sum_{v}[\mp V(v)]$ as given below are the same as in the previous paper [5], but those of $U_{i}$ and $P_{i}(v)$ are different:

$$
\begin{aligned}
f(x) & =\prod_{1}^{\infty}\left(1-x^{n}\right)=\sum_{-\infty}^{+\infty}(-1)^{m} x^{m(3 m+1) / 2}=\sum_{n=0}^{\infty} a_{n} x^{n}, \\
{[f(x)]^{-1} } & =\left[\prod_{1}^{\infty}\left(1-x^{n}\right)\right]^{-1}=\sum_{n=0}^{\infty} p(n) x^{n} . \\
u_{r} & =\sum_{n=0}^{\infty} n^{r} a_{n} x^{n} \sum_{n=0}^{\infty} p(n) x^{n} .
\end{aligned}
$$

$\sum_{v}$ denotes summation over the pentagonal numbers $v$, where 


$$
\begin{gathered}
v=\frac{1}{2} m(3 m+1), \\
\sum_{v}[\mp V(v)]
\end{gathered}
$$

implies that the sign to be prefixed is negative or positive according as $v$ is of the form $(2 m+1)(3 m+2)$ or $m(6 m+1)$. (The first form $(2 m+1)(3 m+2)$ is equivalent to $(2 m+1)(3 m+1)$ as given in the previous paper [5]; $m$ ranges over all integers positive, zero or negative.) It is obvious that

$$
u_{r}=\sum_{v}\left(\mp v^{r} x^{v}\right) / f(x) .
$$

We define the $U_{i}^{\prime}$ 's by

$$
\left\{\begin{array}{l}
U_{0}=2 u_{5}-5 u_{4}+2 u_{3}+5 u_{2}-5 u_{1}+u_{0}, \\
U_{1}=2 u_{5}-3 u_{4}-u_{3}+4 u_{2}-u_{1} \\
U_{2}=2 u_{5}-u_{4}+5 u_{2}+5 u_{1} \\
U_{4}=2 u_{5}+3 u_{4}+3 u_{3}-5 u_{2}-3 u_{1} \\
U_{5}=u_{5}-3 u_{4}-3 u_{3}+4 u_{2}+u_{1} \\
U_{7}=2 u_{5}-2 u_{4}-u_{3}-2 u_{2}+3 u_{1}
\end{array},\right.
$$

We also need polynomials $P_{i}(v)$ in $v$ which like $U_{i}$ are defined only for $i=0,1,2,4,5$ and 7 and which are obtained by replacing $U_{i}$ by $P_{i}(v)$ and $u_{r}$ by $v^{r}$ in the above relations (10).

4. Some lemmas. For the pentagonal numbers $v$ which fall only in the residue classes $i=0,1,2,4,5$ and 7 modulo 11 the following lemma can be verified.

Lemma 1. If $v$ is a pentagonal number, then

$$
\begin{aligned}
P_{i}(v) & \equiv 1 \quad(\bmod 11), \text { if } v \equiv i(\bmod 11) \\
& \equiv 0 \quad(\bmod 11), \text { if } v \neq i(\bmod 11) .
\end{aligned}
$$

Applying relation (9) to (10) we obtain

$$
U_{i}=\sum_{v}\left[\mp P_{i}(v) x^{v}\right] / f(x) ;
$$

and then the use of Lemma 1 leads to Lemma 2.

LEMMA 2. $\quad U_{i} \equiv \sum_{v \equiv i}\left(\mp x^{v}\right) / f(x) \quad(\bmod 11)$,

the summation being extended over all pentagonal numbers $v \equiv i$ $(\bmod 11)$. 
The following lemma can be verified without difficulty by writing $11 m+j$ with $j=0,-4 ;-1,-3 ; 1,-5 ; 3,4 ;-2$; and 2,5 respectively in place of $m$ in the expression $\frac{1}{2} m(3 m+1)$ for the pentagonal numbers, and in $(-1)^{m}$ its associated sign. It is also to be remembered (when $j$ is negative, say, $\left.-j^{\prime}\right)$ that $\frac{1}{2}\left(11 m-j^{\prime}\right)\left(33 m-3 j^{\prime}+1\right)$ and $\frac{1}{2}\left(11 m+j^{\prime}\right)\left(33 m+3 j^{\prime}-1\right)$ represent the same set of numbers.

LEMMA 3. With respect to the modulus 11 the pentagonal numbers $v$ fall in the six residue classes $i=0,1,2,4,5$ and 7; and the solutions of

$$
v \equiv i \quad(\bmod 11)
$$

and the corresponding associated signs are as follows.

\begin{tabular}{|c|c|c|}
\hline$q$ & solutions (1st set): & solutions (2nd set): \\
\hline 0 & $\left.{ }^{2}+11 m\right)$ & ) \\
\hline 1 & $\left.{ }^{2}+55 m\right)+1$ & 2 , ( \\
\hline 2 & $\left.{ }^{2}+77 m\right)+2,(-$ & $n)+35$, $(-$ \\
\hline 4 & $\left.\imath^{2}+209 m\right)+15$ & $m)+26,(-1)^{m}$ \\
\hline 5 & $\frac{1}{2}\left(363 m^{2}+121 m\right)+5,(-1)^{m}$ & \\
\hline 7 & $\frac{1}{2}\left(363 m^{2}+143 m\right)+7,(-1)^{m}$ & $40,(-$ \\
\hline
\end{tabular}

The identities given in the next lemma are simple applications of a special case of a famous identity of Jacobi [2, p. 283] viz.,

$$
\prod_{n=0}^{\infty}\left[\left(1-x^{2 k n+k-l}\right)\left(1-x^{2 k n+k+l}\right)\left(1-x^{2 k n+2 k}\right)\right]=\sum_{-\infty}^{+\infty}(-1)^{m} x^{k m^{2}+l m} .
$$

In establishing this lemma $k$ and $l$ are given values in conformity with the expressions quardratic in $m$ given in Lemma 3.

Lemma 4. If $v$ is a pentagonal number then, writing $v \equiv i$ simply for $v \equiv i(\bmod 11)$, we have

$$
\begin{aligned}
\sum_{v \equiv 0}\left(\mp x^{v}\right)= & \prod_{0}^{\infty}\left[\left(1-x^{363 n+176}\right)\left(1-x^{363 n+187}\right)\left(1-x^{363 n+363}\right)\right] \\
& +x^{22} \prod_{0}^{\infty}\left[\left(1-x^{363 n+55}\right)\left(1-x^{363 n+308}\right)\left(1-x^{363 n+363}\right)\right], \\
\sum_{v \equiv 1}\left(\mp x^{v}\right)= & -x \prod_{0}^{\infty}\left[\left(1-x^{363 n+154}\right)\left(1-x^{363 n+209}\right)\left(1-x^{363 n+363}\right)\right] \\
& -x^{12} \prod_{0}^{\infty}\left[\left(1-x^{363 n+88}\right)\left(1-x^{363 n+275}\right)\left(1-x^{363 n+363}\right)\right],
\end{aligned}
$$




$$
\begin{aligned}
\sum_{v \equiv 2}\left(\mp x^{v}\right)= & -x^{2} \prod_{0}^{\infty}\left[\left(1-x^{363 n+143}\right)\left(1-x^{363 n+220}\right)\left(1-x^{363 n+363}\right)\right] \\
& -x^{35} \prod_{0}^{\infty}\left[\left(1-x^{363 n+22}\right)\left(1-x^{363 n+341}\right)\left(1-x^{363 n+363}\right)\right], \\
\sum_{v \equiv 4}\left(\mp x^{v}\right)= & -x^{15} \prod_{0}^{\infty}\left[\left(1-x^{363 n+77}\right)\left(1-x^{363 n+286}\right)\left(1-x^{363 n+363}\right)\right] \\
& +x^{26} \prod_{0}^{\infty}\left[\left(1-x^{363 n+4}\right)\left(1-x^{363 n+319}\right)\left(1-x^{363 n+363}\right)\right], \\
\sum_{v \equiv 5}\left(\mp x^{v}\right)= & x^{5} \prod_{0}^{\infty}\left(1-x^{121 n+121}\right), \\
\sum_{v \equiv 7}\left(\mp x^{v}\right)= & x^{7} \prod_{0}^{\infty}\left[\left(1-x^{362 n+110}\right)\left(1-x^{363 n+253}\right)\left(1-x^{363 n+363}\right)\right] \\
& -x^{40} \prod_{0}^{\infty}\left[\left(1-x^{363 n+11}\right)\left(1-x^{363 n+352}\right)\left(1-x^{363 n+363}\right)\right] .
\end{aligned}
$$

The next lemma is derived from Lemma 2 after the substitution in it of the product expressions for $\sum_{v \equiv i}\left(\mp x^{v}\right)$ as given in Lemma 4. The following fact is to be used in addition.

$$
\begin{aligned}
& \frac{\prod_{n=0}^{\infty}\left[\left(1-x^{363 n+r}\right)\left(1-x^{363 n+363-r}\right)\left(1-x^{363 n+363}\right)\right]}{f(x)} \\
= & \frac{\prod_{n=0}^{\infty}\left[\left(1-x^{363 n+r}\right)\left(1-x^{363 n+363-r}\right)\left(1-x^{363 n+363}\right)\right]}{\left[(1-x)\left(1-x^{2}\right)\left(1-x^{3}\right) \cdots\right]}=\sum_{n=0}^{\infty}{ }^{363} p(n) x^{n} .
\end{aligned}
$$

LEMMA 5. With respect to the modulus 11

$$
\begin{aligned}
& \left.U_{0} \equiv \sum_{n=0}^{\infty} 36376^{p(n}\right) x^{n}+\sum_{n=0}^{\infty} 363 p p(n-22) x^{n}, \\
& U_{1} \equiv-\sum_{n=0}^{\infty} 363 p 4(n-1) x^{n}-\sum_{n=0}^{\infty} 363 p(n-12) x^{n}, \\
& U_{2} \equiv-\sum_{n=0}^{\infty} 363 p\left(n 3^{n} p(n-2) x^{n}-\sum_{n=0}^{\infty} 363 p(n-35) x^{n},\right. \\
& U_{4} \equiv-\sum_{n=0}^{\infty} 36377 p(n-15) x^{n}+\sum_{n=0}^{\infty} 363 p p(n-26) x^{n}, \\
& U_{5} \equiv \sum_{n=0}^{\infty} 121 p(n-5) x^{n}, \\
& U_{7} \equiv \sum_{n=0}^{\infty} 363 p(n-7) x^{n}-\sum_{n=0}^{\infty} 363 p(n-40) x^{n} .
\end{aligned}
$$

We require a set of congruences which are directly derivable from the identities for $u_{r}=u_{r, 0}$ given in [3], for $r=1,2,3,4$ and 5 . These identities express $u_{r}$ 's as linear functions of $\Phi_{a, b}$ 's. By suitable multiplications of both sides of these identities the fractional coefficients appearing in [3] may be made integral. Since we are concerned 
with congruences modulo 11 we have in the following lemma reduced these coefficients with respect to the modulus 11 . For the sake of simplicity we have written $(a, b)$ instead of $\Phi_{a, b}$.

LEMma 6. With respect to the modulus 11 for the congruences we have

$$
\begin{aligned}
u_{0} \equiv & 1 ; \\
u_{1}= & -(0,1) ; \\
u_{2} \equiv & (0,1)+4(1,2)+5(0,3) ; \\
u_{3} \equiv & 2(0,1)+5(1,2)+5(2,3)-2(0,3)-3(1,4)+3(0,5) ; \\
u_{4} \equiv & -5(0,1)-5(1,2)+(2,3)+4(3,4)+2(0,3)-5(1,4) \\
& -2(2,5)+5(0,5)-4(1,6)-3(0,7) ; \\
u_{5} \equiv & -(0,10)-5(1,2)-4(2,3)+(3,4)-(4,5)+2(0,3) \\
& -2(1,4)+5(2,5)+3(3,6)+2(0,5)-(1,6)+3(2,7) \\
& +2(0,7)-5(1,8) .
\end{aligned}
$$

The next lemma is obtained by the substitution of the above values of $u_{r}$ 's in the expressions for $U_{\imath}$ given in (10).

Lemma 7. With respect to the modulus 11

$$
\begin{aligned}
U_{0}-1 & \equiv L_{0} \\
U_{i} & \equiv L_{i}, \quad i=1,2,4,5,7 ;
\end{aligned}
$$

where

$$
\begin{aligned}
L_{\imath}= & A_{1}(1,8)+A_{0}(0,7) \\
& +B_{2}(2,7)+B_{1}(1,6)+B_{0}(0,5) \\
& +C_{3}(3,6)+C_{2}(2,5)+C_{1}(1,4)+C_{0}(0,3) \\
& +D_{4}(4,5)+D_{3}(3,4)+D_{2}(2,3)+D_{1}(1,2)+D_{0}(0,1),
\end{aligned}
$$

the set of coefficients

$$
\left(A_{1}, A_{0} ; B_{2}, B_{1}, B_{0} ; C_{3}, C_{2}, C_{1}, C_{0} ; D_{4}, D_{3}, D_{2}, D_{1}, D_{0}\right)
$$

being respectively

$$
\begin{aligned}
& (1,-3 ;-5,-4,-4 ;-5,-2,4,4 ;-2,4,-3, \quad 1,4) \text {; } \\
& (1,2 ;-5,-1,-3 ;-5, \quad 5, \quad 3,-2 ;-2, \quad 1,-5, \quad 5, \quad 5) \text {; } \\
& (1,-4 ;-5, \quad 2,-1 ;-5, \quad 1, \quad 1, \quad 5 ;-2,-2, \quad 2, \quad 4, \quad 3) \text {; } \\
& (1,-5 ;-5,-3,-5 ;-5, \quad 4, \quad 5, \quad 1 ;-2, \quad 3,-1, \quad 3,-2) \text {; } \\
& (-5, \quad 0 ; \quad 3, \quad 0, \quad 0 ; \quad 3, \quad 0, \quad 0, \quad 0 ;-1, \quad 0, \quad 0, \quad 0, \quad 0) \text {; } \\
& (1,-1 ;-5,-5, \quad 2 ;-5, \quad 3,-2, \quad 3 ;-2, \quad 5,-4,-2, \quad 1) \text {; }
\end{aligned}
$$

for $i=0,1,2,4,5$ and 7 . 
5. The basic theorem. By comparing the coefficients of the two expressions for $U_{i}(\bmod 11)$ given in Lemmas 6 and 7 we obtain the following theorem from which our final conclusions are drawn.

THEOREM 0. The following congruences are true for $n>0$, the modulus being 11.

$$
\begin{aligned}
& { }_{176}^{363} p(n)+{ }^{363} p(n-22) \\
& (1) \equiv(n-3) \sigma_{7}(n)-\left(5 n^{2}+4 n+4\right) \sigma_{5}(n)-\left(5 n^{3}+2 n^{2}-4 n-4\right) \sigma_{3}(n) \\
& -\left(2 n^{4}-4 n^{3}+3 n^{2}-n-4\right) \sigma(n) \text {; } \\
& -{ }_{154}^{363} p(n-1)-{ }_{88}^{363} p(n-12) \\
& (2) \equiv(n+2) \sigma_{7}(n)-\left(5 n^{2}+n+3\right) \sigma_{5}(n)-\left(5 n^{3}-5 n^{2}-3 n+2\right) \sigma_{3}(n) \\
& -\left(2 n^{4}-n^{3}+5 n^{2}-5 n-5\right) \sigma(n) ; \\
& -{ }_{143}^{363} p(n-2)-{ }_{22}^{363} p(n-35) \\
& (3) \equiv(n-4) \sigma_{7}(n)-\left(5 n^{2}-2 n+1\right) \sigma_{5}(n)-\left(5 n^{3}-n^{2}-n-5\right) \sigma_{3}(n) \\
& -\left(2 n^{4}+2 n^{3}-2 n^{2}-4 n-3\right) \sigma(n) \text {; } \\
& -{ }_{77}^{363} p(n-15)+{ }_{44}^{363} p(n-26) \\
& (4) \equiv(n-5) \sigma_{7}(n)-\left(5 n^{2}+3 n+5\right) \sigma_{5}(n)-\left(5 n^{3}-4 n^{2}-5 n-1\right) \sigma_{3}(n) \\
& -\left(2 n^{4}-3 n^{3}-n^{2}-3 n+2\right) \sigma(n) ; \\
& 121_{p(n-5)} \\
& \equiv-5 n \sigma_{7}(n)+3 n^{2} \sigma_{5}(n)+3 n^{3} \sigma_{3}(n)-n^{4} \sigma(n) ; \\
& { }_{110}^{363} p(n-7)-{ }_{11}^{363} p(n-40) \\
& (6) \equiv(n-1) \sigma_{7}(n)-\left(5 n^{2}+5 n-2\right) \sigma_{5}(n)-\left(5 n^{3}-3 n^{2}+2 n-3\right) \sigma_{3}(n) \\
& -\left(2 n^{4}-5 n^{3}+4 n^{2}+2 n-1\right) \sigma(n) .
\end{aligned}
$$

6. Proofs of Theorems 1 and 2. In view of the well-known congruence [9, 1 p. 167]

$$
\sigma_{s}(n) \equiv 0 \quad(\bmod k)
$$

for 'almost all' $n$ for arbitrarily fixed $k$ and odd $s$ it is a straightforward matter to infer Theorem 1 from Theorem 0 .

The first relation of Theorem 2 is also obtained immediately by writing $11 n+11$ for $n$ in the relation (5) of Theorem 0 . The general result enunciated in Theorem 2 actually emanates from the first 
part, and the process of derivation has two stages. In the first stage Ramanujan's congruence modulo 11 for the partition function $p(n)$ is derived from the first relation, and then this derived relation is used in the second stage to establish the general proposition. It easily follows from (13) and (12) that ${ }_{11} \lambda^{2} p(n)$ can be expressed in the (really finite) form,

$$
{ }_{11 \lambda}^{363} p(n)=p(n)+\sum_{n^{\prime}=1}^{\infty} \varepsilon\left(n^{\prime}\right) p\left(n-11 n^{\prime}\right),
$$

where $\varepsilon\left(n^{\prime}\right)=0$ or \pm 1 . For the special case corresponding to $\lambda=11$ we have the fully specified expression,

$$
{ }^{121} p(n)=\sum_{v}[\mp p(n-121 v)] .
$$

Keeping in mind the first relation of Theorem 2, viz.,

$$
{ }^{121} p(11 n+6) \equiv 0 \quad(\bmod 11),
$$

Ramanujan's congruence is seen to be valid by putting successively $n=6,17,28,39, \ldots$ in (16). Thus (17) implies Ramanujan's congruence. Conversely Ramanujan's congruence implies (17) as can be easily seen when $n$ is replaced by $11 n+6$ in (16). Hence Ramanujan's congruence for the unrestricted partition function is equivalent to the congruence (17) for the restricted partition function. To derive the general proposition we merely write $11 n+6$ for $n$ in (15) and make use of Ramanujan's congruence. It can be easily seen that this latter congruence is also equivalent to any particular case of the general preposition.

7. Corollaries of the basic Theorem O. An interesting consequence of the congruences for the restricted partition functions so far established is that these enable us to deduce a certain congruence property of the divisor function $\sigma_{k}(n)$, viz., Lemma 8 , which in its turn helps us to provide further congruences for the restricted partition functions. This lemma however, is also a particular case of a very general theorem established elsewhere [6].

Lemma 8. If $n$ is not a multiple of 11 then

$$
\sigma_{7}(n) \equiv\left(\frac{n}{11}\right) n^{2} \sigma_{3}(n) \quad(\bmod 11)
$$

where $(n / 11)$ is the Legendre symbol. 
The congruence relation of the divisor functions given in Lemma 8 proves useful for the reduction of the basic congruences of the Theorem 0 into neater forms when attention is separately paid to the cases when $n$ is a quadratic non-residue or a residue of 11 . When $n$ is a multiple of 11 this theorem reduces obviously to an elegant form.

COROLlaRY 1. If $n$ is a quadratic non-residue of 11 , then with respect to the modulus 11 ,

$$
\begin{aligned}
& 363 p(n)+{ }_{563}^{363} p(n-22) \\
& 176^{2} \\
\equiv & \left(5 n^{3}+n^{2}+4 n+4\right) \sigma_{3}(n)-\left(2 n^{4}-4 n^{3}+2 n^{2}-n-4\right) \sigma(n),
\end{aligned}
$$

$$
-{ }_{154}^{363} p(n-1)-{ }_{8}^{363} p(n-12)
$$

$$
\equiv\left(5 n^{3}+3 n^{2}+3 n-2\right) \sigma_{3}(n)-\left(2 n^{4}-n^{3}+5 n^{2}-5 n-5\right) \sigma(n) \text {, }
$$

$$
-{ }_{143}^{363} p(n-2)-{ }_{22}^{363} p(n-35)
$$

$$
\begin{aligned}
\equiv & \left(5 n^{3}+5 n^{2}+n+5\right) \sigma_{3}(n)-\left(2 n^{4}+2 n^{3}-2 n^{2}-4 n-3\right) \sigma(n), \\
& -363 p(n-15)+{ }^{363} p(n-26) \\
\equiv & \left(5 n^{3}-2 n^{2}+5 n+1\right) \sigma_{3}(n)-\left(2 n^{4}-3 n^{3}+n^{2}-3 n+2\right) \sigma(n), \\
& 121 p(n-5) \equiv-3 n^{3} \sigma_{3}(n)-n^{4} \sigma(n), \\
& 363 p(n-7)-363 p(n-40) \\
& 110^{3} p\left(1 n^{3}\right) \\
\equiv & \left(5 n^{3}+4 n^{2}-2 n+3\right) \sigma_{3}(n)-\left(2 n^{4}-5 n^{3}+4 n^{2}+2 n-1\right) \sigma(n) .
\end{aligned}
$$

This corollary easily follows from Theorem 0 when use is made of Lemma 8 which enables replacement of the terms involving $\sigma_{7}(n)$ by terms involving $\sigma_{3}(n)$, and of the following relation (18) which makes redundant the terms involving $\sigma_{5}(n)$ :

$$
\sigma_{5}(11 n+i) \equiv 0 \quad(\bmod 11)
$$

when $i$ is a quadratic non-residue of 11 . This congruence is a particular case of a more general relation [7, 4] which holds for any odd prime modulus.

When $n$ is a quadratic residue of 11 there is no scope for using the relation (18) but Lemma 8 can still be used with some advantage, and the result is given in Corollary 2. 
COROLlary 2. If $n$ is a quadratic residue of 11 then with respect to the modulus 11 ,

$$
\begin{aligned}
& { }_{176}^{363} p(n \quad)+{ }_{55}^{363} p(n-22) \\
& \equiv-\left(5 n^{2}+4 n+4\right) \sigma_{5}(n)-\left(4 n^{3}+5 n^{2}-4 n-4\right) \sigma_{3}(n) \\
& -\left(2 n^{4}-4 n^{3}+3 n^{2}-n-4\right) \sigma(n) \text {, } \\
& -{ }_{154}^{363} p(n-1)-{ }_{88}^{363} p(n-12) \\
& \equiv-\left(5 n^{2}+n+3\right) \sigma_{5}(n)-\left(4 n^{2}+4 n^{2}-3 n+2\right) \sigma_{3}(n) \\
& -\left(2 n^{4}-n^{3}+5 n^{2}-5 n-5\right) \sigma(n) \text {, } \\
& -{ }_{143}^{363} p(n-2)-{ }_{22}^{363} p(n-35) \\
& \equiv-\left(5 n^{2}-2 n+1\right) \sigma_{5}(n)-\left(4 n^{3}+3 n^{2}-n-5\right) \sigma_{3}(n) \\
& -\left(2 n^{4}+2 n^{3}-2 n^{2}-4 n-3\right) \sigma(n) \text {. } \\
& -{ }_{77}^{363} p(n-15)+{ }_{44}^{363} p(n-26) \\
& \equiv-\left(5 n^{2}+3 n+5\right) \sigma_{5}(n)-\left(4 n^{3}+n^{2}-5 n-1\right) \sigma_{3}(n) \\
& -\left(2 n^{4}-3 n^{3}+n^{2}-3 n+2\right) \sigma(n), \\
& { }^{121} p(n-5) \\
& \equiv 3 n^{2} \sigma_{5}(n)-2 n^{3} \sigma_{3}(n)-n^{4} \sigma(n), \\
& 363 p(n-7)-{ }_{11}^{363} p(n-40) \\
& \equiv-\left(5 n^{2}+5 n-2\right) \sigma_{5}(n)-\left(4 n^{3}-2 n^{2}+2 n-3\right) \sigma_{3}(n) \\
& -\left(2 n^{4}-5 n^{3}+4 n^{2}+2 n-1\right) \sigma(n) \text {. }
\end{aligned}
$$

Proof of Theorem 3. This theorem follows from the above corollaries. We shall show that the first set of five congruences of Theorem 3 is deducible from Corollary 1 whereas the last set is obtainable from the other corollary.

Eliminating $\sigma_{3}(n)$ between (5) and each of the remaining congruences of Corollary 1 we find that if $n$ is a quadratic non-residue then

$$
\begin{aligned}
& 3 n^{3}\left[\begin{array}{l}
363 \\
176
\end{array} 6^{36}+{ }_{55}^{363} p(n-22)\right] \\
& +\left(5 n^{3}+n^{2}+4 n+4\right)^{121} p(n-5) \\
\equiv- & n^{3}\left(2 n^{2}+n-1\right) \sigma(n) \quad(\bmod 11),
\end{aligned}
$$


(20)

$$
\begin{aligned}
& -3 n^{3}\left[{ }_{154}^{363} p(n-1)+{ }_{88}^{363} p(n-12)\right] \\
& +\left(5 n^{3}+3 n^{2}+3 n-2\right)^{121} p(n-5) \\
& \equiv n^{3}\left(4 n^{2}-5 n+4\right) \sigma(n) \quad(\bmod 11) \text {, }
\end{aligned}
$$

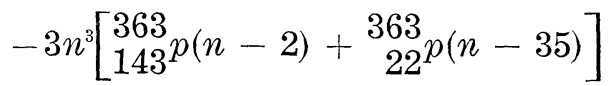

$$
\begin{aligned}
& +\left(5 n^{3}+5 n^{2}+n+5\right)^{121} p(n-5) \\
& \equiv n^{3}\left(5 n^{2}-4 n-2\right) \sigma(n) \quad(\bmod 11) \text {, }
\end{aligned}
$$

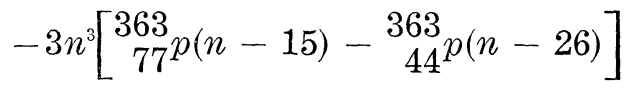

$$
\begin{aligned}
& +\left(5 n^{3}-2 n^{2}+5 n+1\right)^{121} p(n-5) \\
& \equiv n^{3}\left(3 n^{2}-3 n+5\right) \sigma(n) \quad(\bmod 11) \text {, } \\
& 3 n^{3}\left[\begin{array}{l}
363 \\
110
\end{array}(n-7)-{ }^{363} p(n-40)\right] \\
& +\left(5 n^{3}+4 n^{2}-2 n+3\right)^{121} p(n-5) \\
& \equiv n^{3}\left(n^{2}+2 n+3\right) \sigma(n) \quad(\bmod 11) \text {. }
\end{aligned}
$$

By putting $11 n+10,11 n+8,11 n+6,11 n+13$ and $11 n+7$ in place of $n$ in the congruences (19), (20), (21), (22) and (23) respectively we obtain the first five congruences, (1) - (5) of Theorem 3 .

To prove the remaining congruences we turn to Corollary 2. Multiplying both sides of the congruence (5) of this corollary by 5 , and adding the result to each of the other congruences one by one we have respectively the following congruences modulo 11 ,

$$
\begin{aligned}
& { }_{176}^{363} p(n \quad)+{ }_{55}^{363} p(n-22)+5^{121} p(n-5) \\
& \equiv-\left(n^{2}+4 n+4\right) \sigma_{5}(n)-\left(3 n^{3}+5 n^{2}-4 n-4\right) \sigma_{3}(n) \\
& +\left(4 n^{4}+4 n^{3}-3 n^{2}+n+4\right) \sigma(n) \text {, } \\
& -{ }_{154}^{363} p(n-1)-{ }_{88}^{363} p(n-12)+5^{121} p(n-5) \\
& \equiv-\left(n^{2}+n+3\right) \sigma_{5}(n)-\left(3 n^{3}+4 n^{2}-3 n+2\right) \sigma_{3}(n) \\
& +\left(4 n^{4}+n^{3}-5 n^{2}+5 n+5\right) \sigma(n) \text {, } \\
& -{ }_{143}^{363} p(n-2)-{ }_{22}^{363} p(n-35)+5^{121} p(n-5) \\
& \equiv-\left(n^{2}-2 n+1\right) \sigma_{5}(n)-\left(3 n^{3}+3 n^{2}-n-5\right) \sigma_{3}(n) \\
& +\left(4 n^{4}-2 n^{3}+2 n^{2}+4 n+3\right) \sigma(n) \text {, }
\end{aligned}
$$




$$
\begin{aligned}
& \quad-363 p(n-15)+{ }_{44}^{363} p(n-26)+5^{121} p(n-5) \\
& \equiv-\left(n^{2}+3 n+5\right) \sigma_{5}(n)-\left(3 n^{3}+n^{2}-5 n-1\right) \sigma_{3}(n) \\
& \quad+\left(4 n^{4}+3 n^{3}-n^{2}+3 n-2\right) \sigma(n), \\
& 363 p(n-7)-363 p(n-40)+5^{121} p(n-5) \\
& 110^{p} 1^{2}\left(n n^{2}+2 n-3\right) \sigma_{3}(n) \\
& \equiv-\left(n^{2}+5 n-2\right) \sigma_{5}(n)-\left(3 n^{3}-2 n^{2}+2 n+1\right) \sigma(n) .
\end{aligned}
$$

By writing $11 n+9,11 n+5,11 n+12,11 n+15$ and $11 n+14$ respectively in $(24),(25),(26),(27)$ and (28) we obtain the last five congruences $(6)-(10)$ of Theorem 3 .

10. Proof of Theorem 4. This theorem is based upon an artifice which depends upon the following simple congruence which can be established easily from first principles.

$$
\sigma_{k}(11 n) \equiv \sigma_{k}(n) \quad(\bmod 11), k>0 .
$$

We shall illustrate the procedure adopted by proving the last congruence of the theorem. Writing $11 n$ for $n$ in the last congruence (6) of the basic Theorem 0 we have on using the above relation the following

$$
\begin{aligned}
& 363 p(11 n-7)-{ }_{11}^{363} p(11 n-40) \\
& 110 \\
\equiv & -\sigma_{7}(n)+2 \sigma_{5}(n)+3 \sigma_{3}(n)+\sigma(n) \quad(\bmod 11) .
\end{aligned}
$$

Subtracting (6) of Theorem 0 from (30), and then writing $11 n+11$ for $n$ we arrive at the desired result. Other congruences of the theorem are similarly established.

\section{REFERENCES}

1. G. H. Hardy, Ramanujan. Cambridge, 1940.

2. G. H. Hardy and E. M. Wright, An introduction to the theory of numbers, 4th ed. Oxford, 1960.

3. D. B. Lahiri, On a type of series involving the partition function with applications to certain congruence relations, Bull. Calcutta Math. Soc., 38 (1946), 125-132.

4. - On Ramanujan's function $\tau(n)$ and the divisor function $\sigma_{k}(n)-I$. Bull. Calcutta Math Soc., 38 (1946), 193-206.

5. - Some restricted partition functions: congruences modulo 3, Pacific J., Math., 28 (1969), 575-581.

6. - Some congruences for the elementary divisor functions, Amer. Math. Monthly, 76 (1969). 395-397.

7. K. G. Ramanathan, Congruence properties of Ramanujan's function $\tau(n) I I$, J. Indian Math. Soc., 9 (1945), 55-59. 
8. S. Ramanujan, Collected Papers (Cambridge University Press: 1927, and New York, Chelsea Publishing Company: 1962).

9. G. N. Watson, Über Ramanujansche Kongruenzeigenschaften der Zerfällungsanzahlen, Math. Z. 39 (1935) 712-731.

Received July 15, 1969 and in revised form May 26. 1971.

Indian Statistical Institute

Calcutta 


\section{PACIFIC JOURNAL OF MATHEMATICS}

\section{EDITORS}

H. SAMELSON

Stanford University

Stanford, California 94305

C. R. Hовву

University of Washington

Seattle, Washington 98105
J. DUGUNDJI

Department of Mathematics

University of Southern California

Los Angeles, California 90007

RICHARD ARENS

University of California

Los Angeles, California 90024

\section{ASSOCIATE EDITORS}
E. F. BECKENBACH
B. H. NeumanN
F. WOLF
K. YoSHIDA

\section{SUPPORTING INSTITUTIONS}

UNIVERSITY OF BRITISH COLUMBIA

CALIFORNIA INSTITUTE OF TECHNOLOGY

UNIVERSITY OF CALIFORNIA

MONTANA STATE UNIVERSITY

UNIVERSITY OF NEVADA

NEW MEXICO STATE UNIVERSITY

OREGON STATE UNIVERSITY

UNIVERSITY OF OREGON

OSAKA UNIVERSITY

UNIVERSITY OF SOUTHERN CALIFORNIA
STANFORD UNIVERSITY

UNIVERSITY OF TOKYO

UNIVERSITY OF UTAH

WASHINGTON STATE UNIVERSITY

UNIVERSITY OF WASHINGTON

AMERICAN MATHEMATICAL SOCIETY CHEVRON RESEARCH CORPORATION NAVAL WEAPONS CENTER 


\section{Pacific Journal of Mathematics}

\section{Vol. 38, No. $1 \quad$ March, 1971}

Bruce Alan Barnes, Banach algebras which are ideals in a Banach algebra ..... 1

David W. Boyd, Inequalities for positive integral operators............... 9

Lawrence Gerald Brown, Note on the open mapping theorem .............. 25

Stephen Daniel Comer, Representations by algebras of sections over Boolean

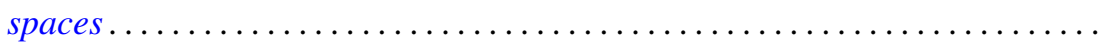

John R. Edwards and Stanley G. Wayment, On the nonequivalence of

conservative Hausdorff methods and Hausdorff moment sequences ........

P. D. T. A. Elliott, On the limiting distribution of additive functions $(\bmod 1) \ldots \ldots$

Mary Rodriguez Embry, Classifying special operators by means of subsets

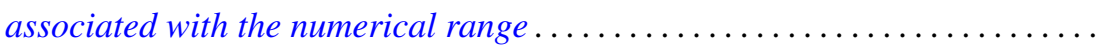

Darald Joe Hartfiel, Counterexamples to a conjecture of G. N. de Oliveira ......

C. Ward Henson, A family of countable homogeneous graphs...............

Satoru Igari and Shigehiko Kuratsubo, A sufficient condition for

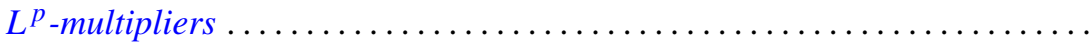

William A. Kirk, Fixed point theorems for nonlinear nonexpansive and

generalized contraction mappings............................

Erwin Kleinfeld, A generalization of commutative and associative rings ...... 95

D. B. Lahiri, Some restricted partition functions. Congruences modulo $11 \ldots \ldots 103$

T. Y. Lin, Homological algebra of stable homotopy ring $\pi *$ of spheres ....... 117

Morris Marden, A representation for the logarithmic derivative of a meromorphic function...........................

John Charles Nichols and James C. Smith, Examples concerning sum properties for metric-dependent dimension functions . .

Asit Baran Raha, On completely Hausdorff-completion of a completely

Hausdorff space.

M. Rajagopalan and Bertram Manuel Schreiber, Ergodic automorphisms and affine transformations of locally compact groups..........

N. V. Rao and Ashoke Kumar Roy, Linear isometries of some function

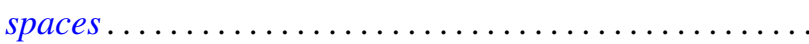

William Francis Reynolds, Blocks and F-class algebras of finite groups

Richard Rochberg, Which linear maps of the disk algebra are multiplicative ...

Gary Sampson, Sharp estimates of convolution transforms in terms of decreasing

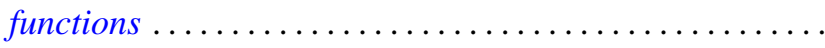

Stephen Scheinberg, Fatou's lemma in normed linear spaces

Ken Shaw, Whittaker constants for entire functions of several complex

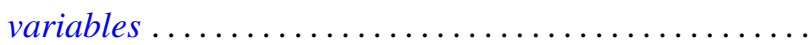

James DeWitt Stein, Two uniform boundedness theorems................ 251

$\mathrm{Li} \mathrm{Pi} \mathrm{Su,} \mathrm{Homomorphisms} \mathrm{of} \mathrm{near-rings} \mathrm{of} \mathrm{continuous} \mathrm{functions} \mathrm{.} \mathrm{.............} 261$

Stephen Willard, Functionally compact spaces, $C$-compact spaces and mappings of minimal Hausdorff spaces....................... 